\title{
INMIGRACIÓN Y EDUCACIÓN. ¿INFLUYE EL NIVEL EDUCATIVO DE LOS PADRES EN EL RENDIMIENTO ACADÉMICO DE LOS HIJOS?
}

\author{
Immigration and school. Does the parents' education level \\ influence their children's school performance?
}

\section{Immigration et école. Le niveau éducatif des parents, ça influe sur les performances scolaires des enfants?}

Mar Lorenzo Moledo*, Miguel Á. Santos RegO** y Agustín Godás Otero****

* Facultad de Ciencias de la Educación. Campus Vida. Universidad de Santiago de Compostela. 15782 Santiago de Compostela.Correo-e: mdelmar.lorenzo@usc.es * Facultad de Ciencias de la Educación. Campus Vida. Universidad de Santiago de Compostela.15782 Santiago de Compostela.Correo-e: miguelangel.santos@usc.es **** Facultad de Psicología. Campus Vida. Universidad de Santiago de Compostela. 15782 Santiago de Compostela.Correo-e: agustin.godas@usc.es

Fecha de recepción: marzo de 2012

Fecha de aceptación definitiva: julio de 2012

Biblid [(1130-3743) 24, 2-2012, 129-148]

RESUMEN

El estudio forma parte de una amplia investigación llevada a cabo en centros educativos de Galicia con el propósito de analizar las variables (familiares, escolares e individuales) que influyen en el rendimiento escolar de los alumnos. Debido al amplio número de variables que hemos manejado, lo que hacemos en este artículo es examinar la relación entre nivel educativo de los padres y rendimiento educativo de sus hijos e hijas, cuestión a la que se está concediendo gran importancia en el panorama internacional (valga como ejemplo el reconocido Informe PISA). 
Concretamente, analizamos el rendimiento académico del alumnado autóctono e inmigrante en las escuelas gallegas, a partir de las calificaciones en cuatro materias. Los resultados muestran la existencia de diferencias entre los dos grupos, pero estas no son estadísticamente significativas. Asimismo, también hemos podido constatar la incidencia que sobre el rendimiento del alumno tiene el nivel de estudios del padre y de la madre, por más que ese efecto se refleje de modo más contundente entre los autóctonos.

Palabras clave: rendimiento académico, PISA, alumnado inmigrante, alumnado autóctono, nivel educativo de las familias.

\section{SUMMARY}

The study is part of an extensive research conducted in Galician education centres with the aim of analysing the variables involved (concerning family, school and individual) that have an influence on pupils' school performance. Due to the large number of variables that we have handled, this paper is focused on examining the relationship between parents' educational level and their children's school performance, an issue that has gained great importance on the international scene (in this sense, it is worth mentioning the famous PISA Report). More specifically, we have analysed the academic performance of native and immigrant pupils in Galician schools, considering their grades in four subjects. The results show the existence of differences between the two groups, but these are not statistically significant. Moreover, we have also noted the impact that parents' educational level has on pupils' performance, although this effect is more obvious among native children.

Key words: performance, school, PISA, immigrant pupils, native pupils, families' education level.

\section{SOMMAIRE}

L'étude fait partie d'une investigation plus étendue réalisée dans les centres d'enseignement de Galicie avec l'objectif d'analyser les variables (de la famille, de l'école et individuelles) qui influent sur les performances scolaires des élèves à l'école. À cause des nombreuses variables utilisées, ce qu'on fait dans cet article c'est d'examiner la relation entre le niveau éducatif des parents et les performances scolaires de ses fils ou filles à l'école; c'est une question qui a pris beaucoup d'importance sur le plan international (on prend comme exemple le fameux Rapport PISA). Concrètement, on analyse le rendement académique des élèves autochtones et immigrés dans les écoles galiciennes, sur la base des notes reçues dans quatre matières. Les résultats montrent une différence entre les deux groupes, mais elle n'est pas significative du point de vue statistique. De la même façon, on a pu constater l'incidence du niveau éducatif du père ou de la mère sur les performances scolaires de l'élève, toutefois que cet effet se montre plus fortement entre les élèves autochtones.

Mots clés: performance scolaire, PISA, ensemble des élèves immigrés, ensemble des élèves autochtones, niveau éducatif des familles. 
1. IntRoducción. El RENDimiento ACADÉmiCo DEL ALUMNADO INMIGRANTE EN ESPAÑA

El rendimiento de los estudiantes está influido por las circunstancias en las que se desarrollan los aprendizajes, los condicionantes de partida, los entornos sociales, económicos y culturales de alumnos y centros educativos (medida de equidad) (Instituto de Evaluación, 2010a; Suárez-Orozco y Suárez-Orozco, 2008). Diversos estudios a nivel nacional e internacional advierten que el grado de adquisición de las competencias básicas por el alumnado tiene relación con el estatus social, económico y cultural de las familias (ver European Commission, 2008).

Utilizando el índice "estatus social, económico y cultural" (ESCS), compuesto por el nivel más alto de estudios de los padres, la profesión, el número de libros en el domicilio familiar y el nivel de recursos domésticos, para explicar la relación entre los resultados de los alumnos de 15 años y el nivel socioeconómico y cultural de las familias, PISA-2009 reitera que cuando se considera la variable "nivel de estudios de los padres" las diferencias en los resultados son importantes y llegan a alcanzar en España casi los 100 puntos y en la OCDE los 120. Esto es, a mayor nivel educativo de los padres mejora la puntuación media de los alumnos. Serra y Palaudàrias (2009) consideran que entre los factores que inciden en las trayectorias de continuidad académica de los alumnos está el nivel de estudios de los padres y, sobre todo, de las madres.

En la misma línea, en un estudio sobre los factores determinantes del rendimiento educativo en Cataluña se llega a la conclusión de que la educación de los padres es un elemento esencial para el proceso de aprendizaje de los más jóvenes (Gutiérrez-Domènech, 2009). Por su parte, Dronkers (2010) afirma, al estudiar la influencia de los países de origen y de destino en el rendimiento educativo del alumnado de origen inmigrante, que las características individuales son mucho más importantes que cualquier (macro)característica para explicar los resultados educativos, y destaca, de forma específica, la educación de los padres y el hecho de proceder de un país con muchos años de educación obligatoria.

Otra de las variables que se incluye en PISA es justamente el origen inmigrante del alumno, llegando a concluirse que la condición de inmigrante incrementa el riesgo de fracaso escolar. Calero, Choi y Waisgrais (2010) analizan a partir de los datos de 2006 las variables que determinan que un alumno se sitúe en el grupo de riesgo de fracaso escolar. Concretamente, estudian factores individuales como el género o la repetición de curso; familiares, entre los que destacan la categoría socioeconómica, la situación laboral o el nivel educativo de los padres, el origen inmigrante de la familia o la lengua hablada en el hogar; o variables de centro como las características de la escuela, sus recursos, los procesos educativos o la composición del alumnado.

Siguiendo a los mismos autores, la probabilidad de que los alumnos de origen inmigrante nacidos en el extranjero (primera generación) se sitúen en el nivel 1 o inferior de PISA duplica a la de los alumnos nacionales (ver también Gutiérrez- 
Domènech, 2009). Por el contrario, no se aprecian diferencias entre los alumnos de familias de origen inmigrante, pero que nacieron en España (segunda generación), y los nacionales. Respecto del nivel de estudios de los padres, concluyen que tiene un efecto no significativo sobre el riesgo de fracaso escolar, aclarando que es posible que su efecto sea absorbido por otras variables correlacionadas, como es el caso de la situación económica, la categoría socioprofesional o el capital cultural.

Una referencia que nos ayudaría a explicar el hecho de encontrar menos diferencias entre autóctonos y alumnos nacidos en España de familias inmigrantes la encontramos en el trabajo de Suárez-Orozco y Suárez-Orozco (2004) con niños y niñas inmigrantes en USA. Afirman estos investigadores de la Universidad de Nueva York que los jóvenes inmigrantes recién llegados de primera generación comparten los mismos retos que los de segunda generación o los de la generación 1,5 (hijos e hijas de familias inmigrantes nacidos en el país de origen, pero que han llegado a la de destino antes de iniciar su proceso de socialización escolar). No obstante, los recién llegados tienen que enfrentarse a los trastornos sociales y culturales inherentes a todo proceso migratorio y, además, adquirir una lengua nueva. Por otro lado, sus padres inmigrantes, con conocimientos escasos de inglés, suelen mostrar dificultades para controlar el progreso escolar de sus hijos, seguir las actividades extraescolares o comprender sus experiencias.

También encontramos en los Informes PISA un aspecto que nos interesa especialmente. Nos referimos a la constatación derivada de que en nuestro sistema educativo hay diferencias en los resultados medios de los alumnos en el nivel autonómico. Fue en el estudio realizado a partir de los datos del 2006 cuando algunas comunidades autónomas salieron a la palestra, permitiendo de ese modo hacer visible una situación que resultó desigual en cuanto a la media de la OCDE (en torno a los 500 puntos). En el caso de ciencias, por ejemplo, este hecho es bien sintomático: Castilla y León (520), La Rioja (520), Aragón (513), Navarra (511), Cantabria (509), Asturias (508), Galicia (505), País Vasco (495) y Cataluña (491). Andalucía y el conjunto de España estaban, sin embargo, por debajo de la media ponderada (cfr. Calero, Choi y Waisgrais, 2009; Cebolla, 2009).

Justamente, la presencia de alumnado extranjero en las muestras especiales de las comunidades autónomas que se sumaron al PISA-2006 sirvió de excusa en la explicación de unos resultados que distaban de ser los previstos. Aun así, tampoco se puede decir que mejore mucho la puntuación total de las CC. AA. si eliminamos a los inmigrantes de la muestra. En conjunto, los inmigrantes bajan menos de seis puntos el rendimiento en el sistema educativo español (Carabaña, 2008). El cuadro, de todos modos, es muy distinto en algunas partes del país (Ferrer et al., 2008). En Galicia no se producen diferencias sustantivas entre el rendimiento de los alumnos nativos y el de los inmigrantes, siendo éste el mayor para el conjunto de las comunidades, y muy próximo al de los autóctonos de otras. Con estos datos no sería fácil defender la existencia de un sistema educativo polarizado en esta comunidad autónoma. Porque, como es posible consultar en Pedró (2009), a propósito de los 
países de la OCDE, es precisamente la comparación entre ellos lo que nos dice que la segregación se asocia, en alto grado, con un mayor diferencial entre los resultados obtenidos por los alumnos migrantes y por los nativos.

TABla 1. Medias de los alumnos nativos E INMigRANTES Y DIFERENCIAS ENTRE ELLAS (CC. AA. ESPAÑOLAS; CIENCIAS; PISA-2006)

\begin{tabular}{|c|c|c|c|c|c|c|}
\hline \multirow[t]{2}{*}{ CC. AA. } & $\begin{array}{c}\text { TODOS } \\
\text { ALUMNOS }\end{array}$ & $\begin{array}{c}\text { SOLO } \\
\text { NATIVOS }\end{array}$ & $\begin{array}{c}\text { SOLO } \\
\text { INMIGRANTES }\end{array}$ & $\begin{array}{c}\text { DIFERENCIA } \\
\text { TODOS ALUMNOS } \\
\text { Y NATIVOS } \\
\end{array}$ & \multirow[t]{2}{*}{$\begin{array}{l}\text { PORCENTAJE } \\
\text { INMIGRANTES }\end{array}$} & \multirow[t]{2}{*}{$\begin{array}{c}\text { DIFERENCIA } \\
\text { NATIVOS E } \\
\text { INMIGRANTES }\end{array}$} \\
\hline & MEDIA & MEDIA & MEDIA & MediA & & \\
\hline Andalucía & 474 & 475 & 429 & -1 & 3 & 46 \\
\hline Aragón & 513 & 518 & 434 & -5 & 6 & 84 \\
\hline Asturias & 508 & 511 & 471 & -2 & 4 & 39 \\
\hline Cantabria & 509 & 513 & 453 & -4 & 5 & 60 \\
\hline Cast. y León & 520 & 522 & 453 & -2 & 3 & 69 \\
\hline Cataluña & 491 & 499 & 428 & -8 & 11 & 72 \\
\hline Galicia & 505 & 506 & 473 & -2 & 3 & 33 \\
\hline La Rioja & 520 & 526 & 447 & -6 & 7 & 79 \\
\hline Navarra & 511 & 515 & 469 & -4 & 8 & 46 \\
\hline País Vasco & 495 & 499 & 426 & -4 & 5 & 72 \\
\hline Otras & 484 & 491 & 433 & -8 & 12 & 58 \\
\hline España & 488 & 494 & 434 & -6 & 8 & 60 \\
\hline
\end{tabular}

Fuente: Tomado de Carabaña, 2008, 5.

Como adelantábamos, son claras las diferencias en cuanto a la desventaja de los hijos de inmigrantes. Un hecho interesante es que no se da la misma representación de las CC. AA. si la ordenación se realiza por notas medias del conjunto del alumnado o desagregando las calificaciones de los hijos de los inmigrantes, en cuyo caso Galicia es la mejor situada; esto es, la distancia entre alumnos inmigrantes y autóctonos es aquí bastante más pequeña. Al mismo tiempo, según sea la materia evaluada tenemos diferencias significativas en los territorios. Mientras que en ciencias las diferencias son menores que en matemáticas, en lengua son las comunidades catalana, aragonesa y riojana las que mayor distancia presentan entre hijos de autóctonos e inmigrantes.

A la hora de buscar explicación a la variabilidad interterritorial creemos que una clave es la composición de los colectivos con mayor presencia en cada parte del Estado. La investigación pone de manifiesto que el peor rendimiento medio es el de los estudiantes de origen africano y el mejor el de los que tienen procedencia asiática (ver Suárez-Orozco y Suárez-Orozco, 2008). Y otra razón a esgrimir en esa línea, máxime viendo las diferencias de posición en PISA cuando se marca "todos los alumnos" respecto de "sólo alumnos de origen inmigrante", apunta a las políticas públicas de cada localización autonómica y a los trazos de su implicación en el cuidado de las condiciones sociales y escolares (ver CIDE, 2005). 
TABla 2. Resultados De los ALumNOS EN PISA-2006

\begin{tabular}{|c|c|c|c|c|c|c|c|c|c|}
\hline \multirow[b]{2}{*}{ CC. AA. ${ }^{1}$} & \multicolumn{2}{|c|}{ MEDIA CIENCIAS } & \multicolumn{2}{|c|}{$\begin{array}{c}\text { MEDIA } \\
\text { MATEMÁTICAS }\end{array}$} & \multicolumn{2}{|c|}{ MEDIA LENGUA } & \multicolumn{3}{|c|}{$\begin{array}{c}\text { \% EMPADRONADOS } \\
\text { MENORES } 15 \text { AÑOS SOBRE } \\
\text { TOTAL INMIGRANTES }\end{array}$} \\
\hline & GENERAL $^{2}$ & INMIGRA. & GENERAL & INMIGRA. & GENERAL & INMIGRA. & UE-25 & ÁFRICA & AMÉRICA \\
\hline Galicia & 7 & 1 & 8 & 1 & 5 & 3 & 4,5 & 4,9 & 53,8 \\
\hline Andalucía & 10 & 2 & 10 & 2 & 10 & 1 & 30,4 & 16,3 & 34,8 \\
\hline Asturias & 6 & 3 & 7 & 4 & 7 & 2 & 1,0 & 5,1 & 67,0 \\
\hline Navarra & 4 & 4 & 3 & 5 & 4 & 4 & 1,3 & 12,7 & 62,5 \\
\hline Cantabria & 5 & 5 & 5 & 3 & 9 & 5 & 0,6 & 4,1 & 60,4 \\
\hline Cas. y León & 1 & 6 & 2 & 9 & 6 & 6 & 3,1 & 10,3 & 44,4 \\
\hline Cataluña & 9 & 7 & 9 & 6 & 8 & 10 & 18,0 & 21,4 & 46,9 \\
\hline P. Vasco & 8 & 8 & 6 & 10 & 2 & 7 & 2,7 & 10,0 & 56,6 \\
\hline La Rioja & 2 & 9 & 1 & 7 & 1 & 8 & 0,5 & 17,0 & 46,7 \\
\hline Aragón & 3 & 10 & 4 & 8 & 3 & 9 & 1,8 & 15,5 & 42,0 \\
\hline
\end{tabular}

Fuente: Tomado de CeBolla, 2009, 34.

Lo que es manifiesto en la tabla es que las condiciones de equidad que vienen dadas por la distancia relativa entre alumnos inmigrantes y autóctonos distan de ser idénticas en el mapa autonómico del país. El caso de Galicia apenas ofrece dudas en este sentido puesto que en las tres materias objeto de evaluación arroja posiciones de liderazgo en la minoración de las distancias entre ambos grupos de alumnos en las escuelas.

Atendiendo a los Informes internacionales y a la investigación en el campo, el Grupo de Investigación Esculca ${ }^{3}$ llevó a cabo un estudio ${ }^{4}$ con dos objetivos claves. De un lado, estudiar el rendimiento escolar de los niños y niñas procedentes de la inmigración en el último ciclo de Educación Primaria y en el $1 .{ }^{\text {er }}$ y $2 .{ }^{\circ}$ curso de Educación Secundaria Obligatoria en las escuelas de Galicia, comparándolo con el de sus compañeros y compañeras de la población autóctona; y de otro, analizar las causas y factores que mayor incidencia pueden tener en el rendimiento escolar de los hijos/as de familias inmigrantes considerando variables culturales, sociales y

1. Solo se incluyen las CC. AA. con muestra representativa en el estudio PISA-2006.

2. La media general es la posición que la CA ocupa según sea la media de todos los estudiantes. La de los inmigrantes resume la distancia entre la media de los nativos y los hijos de los inmigrantes. Ambas están ordenadas de mayor a menor.

3. Grupo de Investigación de la usc reconocido como Unidad Competitiva del Sistema Galego de I+D+I (Orde do 1 de xullo de 2008, DOG 11/07/08) (www.usc.es/esculca).

4. La investigación llevada a cabo tiene por título O rendemento escolar do alumnado procedente da inmigración en Galicia. Un programa de optimización para familias e centros de ensino. Proyecto de convocatoria competitiva de la Dirección Xeral de Investigación e Desenvolvemento (PGIDT-PXI)-Xunta de Galicia (PGIDIT 07SEC009214PR). 
aquellas ligadas tanto a la efectividad del centro escolar al que asisten como a los rasgos individuales e interpersonales del alumno/a.

La perspectiva que adoptamos para analizar el papel de las familias sobre el rendimiento tiene un carácter interactivo por dos razones obvias: de un lado, porque hay dos figuras protagonistas, en la mayoría de las situaciones, que configuran un grupo primario y básico a la hora de desplegar comportamientos que fomenten o inhiban en sus hijos/as los deseos para obtener, en este caso, logros académicos; y de otro, porque, como tal grupo, consigue sus efectos tanto en la dinámica de las relaciones intergrupales, como en las que mantienen con el centro escolar y la propia comunidad en la que viven. Sabemos que esto sucede desde que los niños/as tienen muy poca edad. En realidad, cuando se habla de "educación para el logro" se hace referencia al hecho de animar a los niños a hacer las cosas bien; es decir, a satisfacer o superar criterios elevados cuando tratan de alcanzar objetivos diversos (Shaffer, 2002).

El modelo que hemos propuesto para las familias establece que, a partir de una situación vital con elementos más o menos estables (a los que llamamos «biográficos"), padre y madre encaran una relación con el centro escolar al que acuden los hijos, el cual en nuestro análisis juega un papel de "mediador" debidamente fundamentado, que -a su vez- hace valorar de manera positiva o negativa su satisfacción con el rendimiento de los hijos y con la relación que mantienen con los miembros de la comunidad educativa. A partir de aquí suponemos que, según sea la dirección y el peso que adoptan estas dimensiones (obtenidas a partir de los autoinformes de padres y madres), así será la fuerza y el perfil de su incidencia sobre el rendimiento académico de los hijos/as.

Figura 1. PlanteAmiento GENERAL DEL ESTUDiO REFERIDO A LAS FAMILIAS

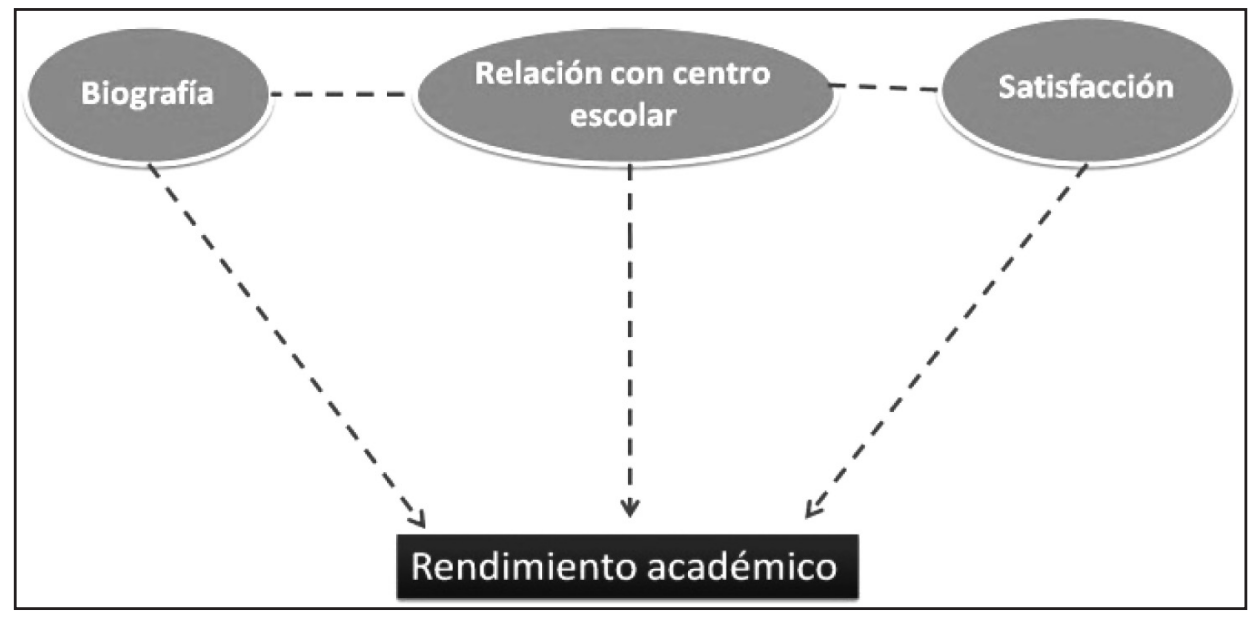


En cuanto a la primera dimensión, que denominamos "biográfica", para así destacar su relativa estabilidad en el conjunto de las influencias que hemos establecido con el resto de los factores, ha sido realizada a partir de las investigaciones desarrolladas por el INCE (1968-1997). Las variables seleccionadas abarcan a ambos padres (edad, estado civil, composición actual del hogar, número de hijos, nacimiento de los hijos en España, nivel de estudios, uso de las lenguas oficiales de la cA en el ámbito familiar, actividad laboral actual, valoración de su situación económica y estructura de valores), a sus hijos e hijas (edad de inicio en la escuela, cambios de centro, y acontecimientos importantes que les hayan afectado en el último año) y, finalmente, a las familias que son inmigrantes, además de las ya expuestas. En relación a esa variable biográfica también se ha recabado información sobre los años de residencia en España y los años que llevan viviendo sus hijos aquí, junto al nivel de dominio del gallego y del castellano.

Justamente, dada la amplitud de variables que hemos manejado, en este trabajo nos hemos detenido en la relación entre nivel educativo de los padres y rendimiento educativo de sus hijos e hijas. Concretamente, hemos analizado el rendimiento académico del alumnado autóctono e inmigrante en las escuelas gallegas, a partir de las calificaciones en cuatro materias, para comprobar si existen diferencias significativas entre ambos grupos y examinar el modo en que se relaciona dicho rendimiento académico con el nivel de estudios del padre y de la madre.

\section{Metodología}

\subsection{Variables de estudio}

Emplearemos como variables de estudio el nivel educativo del padre y de la madre, diferenciando entre autóctonos e inmigrantes, y las notas de sus hijos en Conocimiento del Medio (Primaria) o Ciencias de la Naturaleza (ESO), Lengua Castellana y Literatura, Lengua Gallega y Literatura, y Matemáticas.

\subsection{Población y muestra}

En la determinación de la muestra procedimos a través de un muestreo polietápico, no probabilístico intencional para la selección de las unidades de primer nivel (en este caso los centros donde llevar a cabo el estudio) y también para la selección de las unidades de segundo nivel (individuos).

A fin de seleccionar los elementos de primer nivel, partimos de la explotación de las estadísticas oficiales de la Consellería de Educación e Ordenación Universitaria para el curso escolar 2006/07 de escolarización de niños/as de la inmigración en Galicia. Los centros eran seleccionados si cumplían dos requisitos: ser un centro con Educación Primaria/Secundaria y tener, al menos, diez alumnos inmigrantes 
matriculados en el tercer ciclo de Educación Primaria (preferentemente 6. ${ }^{\circ}$ curso) o en el $1 .^{\circ}$ y $2 .^{\circ}$ curso de Educación Secundaria Obligatoria.

Del total de centros que forman la red educativa gallega, 187 cumplían los requisitos formulados. Inicialmente seleccionamos 43 centros en toda la comunidad autónoma y para ello tuvimos en cuenta el mayor número de alumnos inmigrantes en los dos últimos años de Primaria y en primero y segundo curso de la ESO $^{5}$. Finalmente, el número de centros se quedó en 33 (18 de Educación Primaria y 15 de la ESO).

En definitiva, la muestra total en este segundo nivel está compuesta por un amplio número de familias (madre, padre e hijo/a) y profesores como se refleja en la tabla que sigue.

TABla 3. Muestra de PAdres, madres, ALumnOS y PROFESORES

\begin{tabular}{|c|c|c|c|}
\hline \multirow{2}{*}{ ALUMNOS } & TOTAL & INMIGRANTES & AUTÓCTONOS \\
\cline { 2 - 4 } & 1.401 & 332 & 1.069 \\
\hline \multirow{2}{*}{ PADRES Y MADRES INMIGRANTES } & TOTAL & PADRES & MADRES \\
\cline { 2 - 4 } & 413 & 174 & 239 \\
\hline \multirow{2}{*}{ PADRES Y MADRES AUTÓCTONOS } & TOTAL & PADRES & MADRES \\
\cline { 2 - 4 } & 1.838 & 858 & 980 \\
\hline \multirow{2}{*}{ PROFESORES } & TOTAL & PRIMARIA & ESO \\
\cline { 2 - 4 } & 156 & 37 & 119 \\
\hline
\end{tabular}

\subsection{Características de la muestra}

En la muestra de familias autóctonas hay una mayor presencia de padres y madres de alumnos/as de Primaria $(57,8 \%)$ que de Secundaria Obligatoria. Los progenitores tienen, de forma mayoritaria (64\%), edades comprendidas en el intervalo 36 a 45 años, siendo pocos los que superan (21,4\%) o se sitúan por debajo (14,4\%) de esta franja. Las madres son asalariadas en empresas, amas de casa, o bien trabajan en la administración pública; por su parte, los padres son fundamentalmente empleados de empresas o autónomos.

El número de hijos es de dos (61,9\%), siendo escasas las familias numerosas (19,3\%). Los niños nacieron, como era previsible, en España salvo un porcentaje muy pequeño $(4,8 \%)$ que lo hizo fuera de nuestro país. Su escolarización se inició entre los 2 y los 4 años (72,6\%), e incluso el 24\% empezaron antes de los dos. Desde que iniciaron la educación obligatoria no cambiaron de centro y cuando

5. Tengamos en cuenta que las estadísticas nos proporcionaron el número total de alumnos en Primaria y en los dos primeros cursos de la Secundaria Obligatoria, por lo que tuvimos que indagar centro a centro donde teníamos alumnado matriculado en los cursos que interesaban para el estudio. 
lo hicieron, tal hecho se redujo a una sola ocasión (37,9\%). Únicamente el 24,8\% repitieron algún curso.

Por otro lado, el 53,2\% de los padres inmigrantes tiene a su hijo/a escolarizado en Educación Primaria y el 46,8\% restante son los progenitores de un alumno/a de Secundaria Obligatoria. Se trata de adultos entre los 36 y 45 años $(53,8 \%)$, aunque un porcentaje importante se sitúa en el intervalo inmediatamente inferior $(28,7 \%)$. Las madres declaran en el 27,8\% de los casos ser amas de casa, y las restantes trabajan en empresas, en el servicio doméstico o en la hostelería. Los padres, con notoria diferencia, trabajan más como empleados en empresas.

Tienen entre dos $(33,2 \%)$ y tres hijos/as $(29,8 \%)$, pero el número de familias numerosas es mayoritario (53,8\%). El 93,5\% de los niños de estas familias no nacieron en el país. Se trata de familias que proceden, fundamentalmente, de América Central y del Sur, a las que siguen las africanas. Al analizar la edad de inicio de la escolarización de los hijos/as, los porcentajes se agrupan en torno a los intervalos 2-3, 3-4 y 4-6, por más que sea importante la que se sitúa en más de 6 años (12,6\%). El $78,1 \%$ de los niños y niñas cambiaron de centro en alguna ocasión, y no lo hizo nunca tan sólo el 21,9\%. El 40,5\% de ellos repitió curso en nuestro país.

Los niños/as, mayoritariamente, comprenden, leen y escriben el gallego, pero únicamente lo hablan el 59,9\%. Prácticamente el 100\% comprende, lee, escribe y habla el castellano.

\subsection{Instrumentos}

Empleamos en la investigación cinco instrumentos, que el equipo diseñó teniendo en cuenta los objetivos y los principales hallazgos científicos en el campo. El primer borrador de estos dispositivos fue sometido a un Grupo de Discusión (ver Krueger, 1991) formado por profesionales y miembros de la comunidad educativa, seleccionados basándonos en su capacidad de análisis respecto a los puntos fuertes y débiles de los instrumentos. Para este artículo y al objeto de medir las variables de estudio nos referiremos a tres de ellos:

a) Escala para padres/madres autóctonos: se trata de una escala en gallego que se compone de 45 preguntas, 15 cerradas y 30 de escala tipo Likert que agrupamos en tres categorías: biografía familiar, mediadores y respuestas.

b) Escala para padres/madres inmigrantes: es una escala en castellano, dadas las dificultades de los progenitores para leer y comprender el gallego. Está formada por 56 preguntas, 17 cerradas, 1 abierta y 38 de escala tipo Likert agrupadas en las mismas categorias.

c) Protocolo del Profesorado de Educación Primaria/Educación Secundaria Obligatoria: se diseñó para registrar las notas (respuestas) de los alumnos en cuatro áreas (Educación Primaria) o materias (Educación Secundaria Obligatoria), elegidas teniendo en cuenta los ámbitos que mide PISA (Lectura, Matemáticas y Ciencia): Conocimiento del Medio (Primaria) 
o Ciencias de la Naturaleza (ESO), Lengua Castellana y Literatura, Lengua Gallega y Literatura, y Matemáticas. Las codificamos en cinco alternativas de respuesta, cada una de ellas (insuficiente, suficiente, bien, notable y sobresaliente) con una puntuación de 1 a 5. Además, recoge para cada alumno si tuvo o tiene adaptaciones curriculares, ayudas para la adquisición de lenguas o refuerzos educativos. Por lo tanto, el criterio para evaluar a los estudiantes de nuestra muestra proviene directamente de los tutores y no del paso de un examen externo como es el caso del "Programme for International Students Assessment" (PISA) (ver GutiérrezDomènech, 2009).

\section{ANÁLISIS DE DATOS Y DISCUSIÓN DE RESULTADOS}

Iniciamos este análisis con el estudio de las calificaciones de los alumnos en las distintas materias. Analizando las notas medias obtenidas por los alumnos inmigrantes en cada una de las materias, observamos que se corresponden con una calificación media de "suficiente". En cambio, si nos centramos en los porcentajes de "insuficientes", y la calificación más frecuente, vemos que se corresponde con esta categoría (un 39,8\% en Conocimiento del Medio/Ciencias de la Naturaleza, un 41,2\% en Lengua y Literatura Castellana, un 39,12\% en Lengua y Literatura Gallega y un 42,2\% en Matemáticas).

\section{GRÁFICA 1. NOTAS MEDIAS EN LAS MATERIAS DE ESTUDIO}

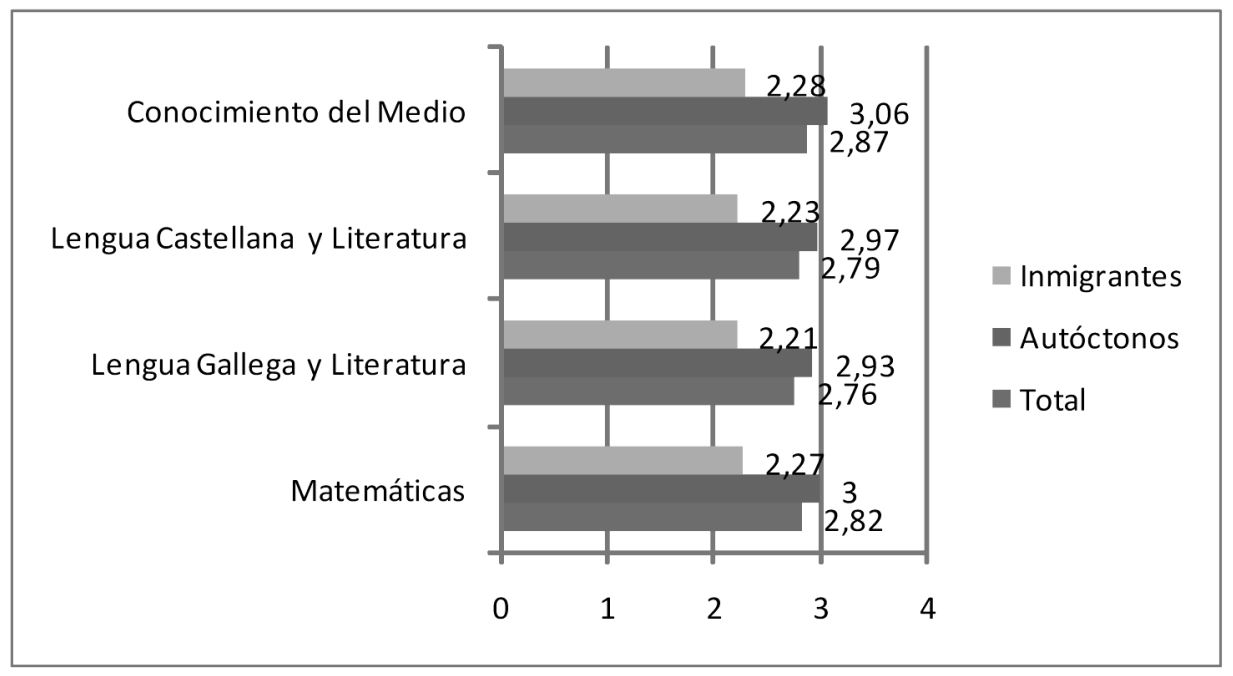


Para los autóctonos, las notas medias en cada materia se corresponden con la categoría de "bien"; en cambio, al analizar los porcentajes y las respuestas más informadas, la calificación que corresponde es la de "notable" (23,2\% en Conocimiento del Medio/Ciencias de la Naturaleza; 24,6\% en Lengua y Literatura Castellana; 24,8\% en Lengua y Literatura Gallega; y un 24,3\% en Matemáticas).

En definitiva, las calificaciones son, en general, ligeramente superiores en el alumnado autóctono en las cuatro materias observadas. A pesar de esta diferencia (notable frente a insuficiente como el registro más frecuente) la dispersión de calificaciones es ligeramente más amplia en el alumnado autóctono y más compacta en el otro grupo.

De forma general, ambos grupos no han realizado adaptaciones curriculares, ni de adquisición de lenguas, ni refuerzo educativo. Las diferencias, en términos porcentuales, son mínimas en los dos primeros aspectos y bastante claras en el refuerzo educativo. No en vano lo ha recibido el 12,7\% del alumnado autóctono, frente al 30,6\% de los estudiantes de origen inmigrante.

Estos matices diferenciales serán ahora contrastados de un modo más formal y preciso. A pesar de esta valoración, y como se puede comprobar en los niveles de significación, no podemos decir, en términos de diferencias intergrupales, que éstas sean significativas, razón que nos lleva a concluir que se trata de grupos académicamente relacionados, esto es, con cualificaciones estadísticamente semejantes.

¿Cómo podemos explicar que no existan diferencias entre los alumnos de ambos grupos, cuando, además, la mayoría del alumnado que denominamos inmigrante no ha nacido en España, dato comprensible ya que estamos hablando de primoinmigrantes, y llevan aquí entre 1 y 6 años (35,4\% entre 1 y 3 años y 32,8\% entre 4 y 6 años)? Si comparamos estos datos con otros estudios que toman a PISA como referencia (ver Calero, Choi y Waisgrais, 2010), deberíamos encontrar una mayor diferencia entre los dos grupos, ya que estamos ante alumnos inmigrantes de primera generación.

¿Cómo explicamos esto cuando el número de repetidores es superior entre el alumnado inmigrante (40,5\% frente a un $24,8 \%$ en la de autóctonos)? En este sentido, los estudios de PISA concluyen que "la repetición y el retraso en el estudio no impiden que los resultados académicos de los alumnos que padecen repetición sean francamente negativos" (Instituto de Evaluación, 2010a, 110). Parece que la repetición de curso es el fenómeno que más negativamente afecta a los resultados de los alumnos españoles, lo que debería suponer un replanteamiento de la utilidad de esta medida, tal y como se materializa en nuestro sistema educativo (ver Fernández Enguita, Mena y Riviere, 2010). Debemos matizar que mientras los porcentajes de repeticiones entre el alumnado inmigrante $(24,4 \%)$ y el autóctono $(27,4 \%)$ son muy próximos en ESO, en Primaria se observan mayores diferencias (67,9\% y $58,6 \%$, respectivamente). 
MAR LORENZO MOLEDO, MIGUEL Á. SANTOS REGO Y AGUSTÍN GODÁS OTERO

INMIGRACIÓN Y EDUCACIÓN. ¿¿INFLUYE EL NIVEL EDUCATIVO DE LOS PADRES EN EL RENDIMIENTO ACADÉMICO DE LOS HIJOS?

TABLA 4. DifERENCIAS INTERGRUPO EN RENDIMIENTO DEL ALUMNADO

\begin{tabular}{|c|c|c|c|c|c|c|c|}
\hline & & & & & & & \\
\hline & & & & & & \multirow{2}{*}{$\begin{array}{c}\text { CALIFICACIONES } \\
\text { AUTÓCTONO } \\
\%\end{array}$} & \multirow{2}{*}{$\begin{array}{c}\text { CALIFICACIONES } \\
\text { INMIGRANTE } \\
\%\end{array}$} \\
\hline$\chi^{2}$ & $\mathrm{gl}$ & $\mathrm{p}$ & $\%$ fe $\leq 5$ & \multicolumn{2}{|c|}{ VARIABLES } & & \\
\hline \multirow{5}{*}{68,121} & \multirow{5}{*}{4} & \multirow{5}{*}{, 000} & \multirow{5}{*}{0} & \multirow{5}{*}{$\begin{array}{c}\text { CONOCIMIENTO } \\
\text { MEDIO/CIENCIAS } \\
\text { NATURALEZA }\end{array}$} & Insuficiente & 21,5 & 39,8 \\
\hline & & & & & Suficiente & 18,4 & 23,2 \\
\hline & & & & & Bien & 14,6 & 15,3 \\
\hline & & & & & Notable & 23,2 & 12,1 \\
\hline & & & & & Sobresaliente & 22,3 & 9,6 \\
\hline \multirow{5}{*}{69,274} & \multirow{5}{*}{4} & \multirow{5}{*}{, 000} & \multirow{5}{*}{0} & \multirow{5}{*}{ L. CAStellana } & Insuficiente & 20,9 & 41,2 \\
\hline & & & & & Suficiente & 20,3 & 22 \\
\hline & & & & & Bien & 17 & 14,7 \\
\hline & & & & & Notable & 24,6 & 16,6 \\
\hline & & & & & Sobresaliente & 17,2 & 5,5 \\
\hline \multirow{5}{*}{72,817} & \multirow{5}{*}{4} & \multirow{5}{*}{, 000} & \multirow{5}{*}{0} & \multirow{5}{*}{ L. GALLEGA } & Insuficiente & 18,4 & 39,1 \\
\hline & & & & & Suficiente & 24,4 & 26,7 \\
\hline & & & & & Bien & 17,5 & 13 \\
\hline & & & & & Notable & 24,8 & 16,3 \\
\hline & & & & & Sobresaliente & 14,9 & 4,9 \\
\hline \multirow{5}{*}{74,229} & \multirow{5}{*}{4} & \multirow{5}{*}{, 000} & \multirow{5}{*}{0} & \multirow{5}{*}{ MATEMÁTICAS } & Insuficiente & 20,9 & 42,2 \\
\hline & & & & & Suficiente & 19,7 & 17,6 \\
\hline & & & & & Bien & 16,4 & 19,2 \\
\hline & & & & & Notable & 24,3 & 13,4 \\
\hline & & & & & Sobresaliente & 18,7 & 7,6 \\
\hline \multirow{2}{*}{79,659} & \multirow{2}{*}{1} & \multirow{2}{*}{, 000} & \multirow{2}{*}{0} & \multirow{2}{*}{$\begin{array}{l}\text { ADAPTACIONES } \\
\text { CURRICULARES }\end{array}$} & Sí & 2,1 & 5,2 \\
\hline & & & & & No & 97,9 & 94,8 \\
\hline \multirow{2}{*}{55,817} & & $\cap \cap 0$ & , & REFUERZO & Sí & 12,7 & 30,6 \\
\hline & 1 & , & 0 & EDUCATIVO & No & 87,3 & 69,4 \\
\hline 2919 & 1 & 088 & 0 & ADQUISICIÓN & Sí & 0,1 & 0,6 \\
\hline 2,7 & 1 & & & LENGUAS & No & 99,9 & 99,4 \\
\hline
\end{tabular}


Quizás una de las razones tengamos que buscarla en la procedencia de las familias de estos alumnos. El 68,9\% de ellas vienen desde países de América Central y del Sur, el 17,5\% de África y el 8,5\% de la uE. Se infiere que la lengua hablada en el hogar es el castellano y los niños y niñas se manejan, por lo tanto, en una de las dos lenguas vehiculares de enseñanza. Un dato que refuerza esta idea es que estos alumnos no han necesitado ir a un grupo de adquisición de lenguas ${ }^{6}(99,4 \%)$. Calero, Choi y Waisgrais (2010) observan que los alumnos de familias de origen inmigrante de segunda generación que no hablan castellano en sus casas tienen una mayor probabilidad de tener un mal resultado en comparación con aquellos nacidos en España pero que hablan castellano u otra lengua oficial en sus hogares.

Además, en el caso de Galicia, tenemos que referirnos específicamente a las variables de centro y, más concretamente, a la concentración de alumnado inmigrante en los centros educativos. Distinguimos en este sentido, siguiendo al Síndic de Greuges (2009), entre la segregación como desigualdad (segregación escolar), el nivel de equidad entre centros escolares en la distribución de los diferentes grupos y la segregación como exposición (concentración escolar), para referirnos al nivel de concentración de un determinado grupo social en las diferentes escuelas. Según Calero, Choi y Waisgrais (2010) la presencia de alumnos de origen inmigrante en los centros tan sólo incrementa el riesgo de fracaso escolar cuando la concentración supera al 20\% del alumnado total. Es lo que se denomina "efecto compañero". Comprobamos esta variable en todos los centros que participaron en la investigación y ninguno supera ese porcentaje (ver Sotelino y Varela, 2011).

De forma general, en otros estudios hemos demostrado que el nivel de segregación es objetivamente bajo entre las escuelas públicas de Galicia, lo que demuestra una distribución más equilibrada del alumnado extranjero (Santos, Crespo, Lorenzo y Godás, 2011). Justamente, en el último informe PISA (Instituto de Evaluación, 2010a) se concluye que el sistema educativo español es, después del finlandés, aquel en el que se observa mayor equidad (ver también Instituto de Evaluación, 2010b). Más concretamente, Galicia y el País Vasco son las comunidades en las que el sistema educativo se muestra más equitativo. En un sistema educativo equitativo el impacto del estatus social, económico y cultural sobre el rendimiento es pequeño; esto es, el éxito educativo de los estudiantes es esencialmente independiente del entorno social, económico y cultural, tanto de su familia como del centro educativo.

Además, Suárez-Orozco y Suárez-Orozco (2008) utilizando una muestra de 309 alumnos inmigrantes (primera generación) de distritos escolares del área metropolitana

6. Según el Decreto 79/2010, do 20 de maio, para o plurilingüismo no ensino non universitario de Galicia "El alumnado que se incorpore al sistema educativo de Galicia en tercer ciclo de educación primaria, en educación secundaria obligatoria o en bachillerato, procedente de otras comunidades autónomas o de un país extranjero, podrán obtener una exención temporal de la calificación de las pruebas de evaluación de la materia de lengua gallega durante un máximo de dos cursos escolares". 
de Boston y de San Francisco llegan a la conclusión de que lo que caracteriza a los alumnos/as con alto rendimiento es, entre otros, estudiar en centros con menor nivel de segregación y tener madres con estudios secundarios.

Para seguir profundizando en estas diferencias analizamos la distribución de las distintas calificaciones en las cuatro materias según el nivel de estudios del padre y de la madre en ambas muestras. Comenzaremos con un análisis descriptivo del nivel educativo de las familias.

El 39,1\% de las familias autóctonas confiesa tener estudios secundarios e, incluso, el 17,1\% universitarios, aunque el mayor porcentaje (42,2\%) se sitúa en los estudios primarios. En todo caso, hay más madres con estudios secundarios y universitarios que padres.

TABLA 5. NIVEL DE ESTUDIOS DE PADRE Y MADRE

\begin{tabular}{|c|c|c|c|c|c|c|}
\cline { 2 - 7 } \multicolumn{1}{c|}{} & \multicolumn{3}{c|}{ AUTÓCTONOS } & \multicolumn{3}{c|}{ INMIGRANTES } \\
\cline { 2 - 7 } \multicolumn{1}{c|}{} & TOTAL & PADRES & MADRES & TOTAL & PADRES & MADRES \\
\hline Sin estudios & 1,7 & 2,1 & 1,2 & 5,4 & 7,2 & 4,2 \\
\hline Primarios & 42,2 & 43,2 & 41,2 & 23 & 24,7 & 21,8 \\
\hline Secundarios & 39,1 & 38,7 & 39,5 & 49,9 & 48,8 & 50,6 \\
\hline Universitarios & 17,1 & 15,9 & 18 & 21,7 & 19,3 & 23,4 \\
\hline
\end{tabular}

Claramente, los padres (68,1\%) y madres (74\%) inmigrantes tienen estudios medios o superiores. Ellas tienen mayor nivel educativo que los padres, entre los que el $7 \%$ carecen de estudios.

Comparando estos datos debemos señalar, en primer lugar, que no existen diferencias entre padres y madres en cuanto a su nivel de estudios, tanto en las familias autóctonas $\left(\chi^{2}=3,278\right.$; g.l. $=3 ; \mathrm{p} \leq, 351$; fe $\left.\leq 0 \%\right)$ como en las inmigrantes $\left(\chi^{2}=2,686\right.$; $\mathrm{p} \leq, 443$; fe $\leq 0 \%$ ). En segundo lugar, como podemos comprobar, el nivel de estudios de los padres y madres inmigrantes es superior al de los autóctonos. Estas diferencias sí son estadísticamente significativas $\left(\chi^{2}=59,759 ; p \leq, 000 ; 0 \% \mathrm{fe}<5\right)$.

TABla 6. Nivel De ESTUdios SEGÚN PROCEDENCIA Y ÁREA SOCIOPOLÍTICA

\begin{tabular}{|c|c|c|c|c|c|}
\hline \multicolumn{6}{|c|}{ NIVEL DE ESTUDIOS } \\
\hline \multirow{4}{*}{ 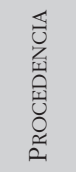 } & & PRIMARIOS & SECUNDARIOS & UNIVERSITARIOS & SIN ESTUDIOS \\
\hline & Autóctonos & 42,2 & 39,1 & 17,1 & 1,7 \\
\hline & Extranjeros & 23,1 & 49,4 & 21,1 & 6,5 \\
\hline & Total & 39,4 & 40,6 & 17,6 & 2,4 \\
\hline \multirow{5}{*}{ 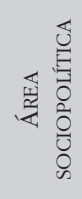 } & Europa & 20,9 & 46,5 & 25,6 & 7,0 \\
\hline & América & 18,3 & 56,6 & 24,4 &, 7 \\
\hline & África & 36,6 & 31,0 & 11,3 & 21,1 \\
\hline & Asia & 63,6 & 18,2 & 9,1 & 9,1 \\
\hline & Total & 23,0 & 50,0 & 21,8 & 5,2 \\
\hline
\end{tabular}


También entre las familias inmigrantes hemos encontrado diferencias estadísticamente significativas en lo que tiene que ver con el nivel de estudios. Las familias de origen americano, mayoritarias en la muestra, e incluso europeo tienen estudios medios o superiores, mientras que las familias procedentes de África son las que menor nivel de estudos poseen, seguidas de las asiáticas $\left(\chi^{2}=77,890\right.$; $p \leq, 000 ; 31,3 \%$ fe $<5$ ).

De forma consistentemente acorde con los objetivos de nuestro trabajo, lo que se aprecian son diferencias en el momento de comparar las calificaciones de los hijos con el nivel de estudios de los padres:

- Cuanto menor es el nivel de estudios de ambos padres, más aumenta el número de "suspensos" en las cuatro materias y más diminuye el número de "notables" y "Sobresalientes". Esta apreciación se observa con más claridad y cuantía en las familias inmigrantes.

- Cuando padres y madres tienen estudios secundarios, aumentan las «buenas" calificaciones de modo considerable, por lo cual las diferencias entre ambas muestras aparecen cuando se constata en las familias inmigrantes un número notablemente menor de tales calificaciones.

- Cuando los estudios son superiores, el aumento de "notables" y "sobresalientes" es importante, sobre todo en las familias autóctonas. En las de origen inmigrante, únicamente se registran semejanzas con las autóctonas en las calificaciones de "bien" y "notable".

- Finalmente, es necesario destacar que las materias donde las diferencias son mayores entre las dos muestras son lengua gallega, en primer lugar, y matemáticas en segundo lugar.

Sin embargo, aunque los datos como vemos en la Tabla 7 demuestren que el nivel educativo de los padres se relaciona con el rendimiento de los más jóvenes, también deberían ser considerados otros factores susceptibles de explicar los peores resultados del alumnado procedente de la inmigración, incluso cuando el nivel de estudios de sus padres es equiparable al de sus compañeros autóctonos. Esas variables (individuales, familiares y escolares) pueden estar ejerciendo un mayor peso en la muestra de alumnado extranjero (ver Santos Rego, 2010). Lo que también se confirma es que las mayores diferencias se dan en dos materias, concretamente lengua gallega y matemáticas. 
MAR LORENZO MOLEDO, MIGUEL Á. SANTOS REGO Y AGUSTÍN GODÁS OTERO

INMIGRACIÓN Y EDUCACIÓN. ¿INFLUYE EL NIVEL EDUCATIVO DE LOS PADRES EN EL RENDIMIENTO ACADÉMICO DE LOS HIJOS?

Tabla 7. Porcentajes en las Calificaciones de los hijos/AS SEGÚN EL NIVEL DE ESTUDIOS DEL PADRE Y DE LA MADRE PARA LAS DOS MUESTRAS ${ }^{7}$

\begin{tabular}{|c|c|c|c|c|c|c|c|c|c|}
\hline & & \multicolumn{2}{|c|}{$\begin{array}{c}\text { CONOCIMIENTO } \\
\text { MEDIO/CIENCIAS } \\
\text { NATURALEZA }\end{array}$} & \multicolumn{2}{|c|}{$\begin{array}{c}\text { LENGUA } \\
\text { CASTELLANA }\end{array}$} & \multicolumn{2}{|c|}{ LENGUA GALLEGA } & \multicolumn{2}{|c|}{ MATEMÁTICAS } \\
\hline & & PADRE & MADRE & PADRE & MADRE & PADRE & MADRE & PADRE & MADRE \\
\hline \multirow{4}{*}{ 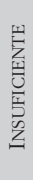 } & Primarios & $2,9 / 48,7^{*}$ & $24,6 / 54,2^{*}$ & $22,3 / 41^{*}$ & $25,1 / 52,4^{*}$ & $21,5 / 43,6^{*}$ & $24,1 / 45,8^{*}$ & $21,8 / 48,7^{*}$ & $24 / 49^{*}$ \\
\hline & Secundarios & $5,2 / 31,2^{*}$ & $18,6 / 34,8^{*}$ & $15,8 / 33,7^{*}$ & $18,6 / 34,8^{*}$ & $13,6 / 31,6^{*}$ & $15,6 / 33^{*}$ & $14,2 / 33,8^{*}$ & $18,2 / 39,3^{*}$ \\
\hline & Superiores & $8,8 / 26,6^{*}$ & $8,2 / 20,7^{*}$ & $6,3 / 26,7^{*}$ & $8,1 / 22,6^{*}$ & $7,1 / 28,6^{*}$ & $5 / 24,5^{*}$ & $6,3 / 40,1^{*}$ & $6,9 / 24,5^{*}$ \\
\hline & Sin estudios & $2,2 / 72,7^{*}$ & $41,7 / 50^{*}$ & $16,7 / 63,6^{*}$ & $25 / 50^{*}$ & $16,7 / 63,6^{*}$ & $41,7 / 50^{*} /$ & $16,7 / 63,6^{*}$ & $50,2 / 60^{*}$ \\
\hline \multirow{4}{*}{ 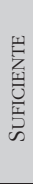 } & Primarios & $, 9 / 17,9$ & $20,4 / 16,7^{*}$ & $8 / 33,3^{*}$ & $22,7 / 25^{*}$ & $23,8 / 25,6^{*}$ & $23,1 / 29$ & $20,9 / 20,5^{*}$ & $21,8 / 18,4^{*}$ \\
\hline & Secundarios & $16,6 / 23,4^{*}$ & $19,4 / 21,4^{*}$ & $15,5 / 19,5^{*}$ & $18,6 / 24,1^{*}$ & $21 / 22,4^{*}$ & $26,9 / 26,7^{*}$ & $17,5 / 13^{*}$ & $19,8 / 17^{*}$ \\
\hline & Superiores & $9,4 / 20^{*}$ & $10,7 / 20,7^{*}$ & $9,4 / 20^{*}$ & $9,4 / 18,9^{*}$ & $11,8 / 32,1^{*}$ & $13,1 / 20,4^{*}$ & $11 / 13,3^{*}$ & $8,8 / 17^{*}$ \\
\hline & Sin estudios & $16,7 / 9,1^{*}$ & $3 / 20^{*} /$ & $27,8 / 9,1^{*}$ & $33,3 / 10^{*}$ & $33,3 / 18,2^{*}$ & $25 / 30^{*}$ & $33,3 / 0^{*}$ & $16,6 / 0^{*}$ \\
\hline \multirow{4}{*}{ 届 } & Primarios & $5,2 / 10,3^{*}$ & $13,5 / 14,6^{*}$ & $17,9 / 2,6^{*}$ & $15 / 6,3^{*}$ & $18,5 / 15,4^{*}$ & $17,8 / 12,5^{*}$ & $19,7 / 10,3^{*}$ & $15,6 / 20,4^{*}$ \\
\hline & Secundarios & $14 / 13$ & $15,6 / 19,6$ & $17,4 / 19,6$ & $19,4 / 13,4$ & $16,5 / 17,1$ & $15,6 / 17,9$ & $16,5 / 24,7$ & $18,4 / 22,3$ \\
\hline & Superiores & $9,4 / 16,7^{*}$ & $10,7 / 18,9^{*}$ & $15 / 16,7^{*}$ & $17,5 / 15,1^{*}$ & $12,6 / 3,6^{*}$ & $16,9 / 18,4^{*}$ & $14,2 / 13,3^{*}$ & $15,6 / 24,5^{*}$ \\
\hline & Sin es & $22,2 / 9,1^{*}$ & $0^{*}$ & $, 2 / 18,2^{*}$ & 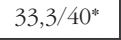 & 1 & 20 & $6 / 18,2^{*}$ & $16,6 / 20^{*}$ \\
\hline \multirow{4}{*}{ 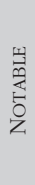 } & Primarios & $8 / 12,8^{*}$ & $24,0 / 0,5$ & * & $*$ & 23,5 & $21, \mathrm{c}$ & $22,4 / 1$ & $2^{*}$ \\
\hline & Secundarios & $24,7 / 20,8^{*}$ & $20,3 / 15,2^{*}$ & $29,7 / 24,7^{*}$ & $24,8 / 23,2^{*}$ & $29,1 / 25^{*}$ & $23,9 / 17,9^{*}$ & $28,2 / 22^{*}$ & $24,6 / 10^{*}$ \\
\hline & Superiores & $29,9 / 16,7^{*}$ & $30,2 / 20,8^{*}$ & $33,9 / 26,6^{*}$ & $31,9 / 32,1^{*}$ & $37,8 / 25^{*}$ & $40,6 / 24,5^{*}$ & $35,4 / 23,3^{*}$ & $29,4 / 18,9^{*}$ \\
\hline & Sin $\mathrm{e}$ & 16,7 & $0^{*}$ & $1^{*}$ & $8,3 / 0^{*}$ & 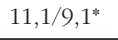 & 8, & 16 & $16,6 / 20^{*}$ \\
\hline \multirow{4}{*}{ 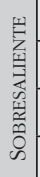 } & Primarios & $16,2 / 10,3^{*}$ & $16,9 / 8,2^{*}$ & $13,4 / 0^{*}$ & 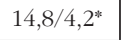 & $2,7 / 0^{*}$ & $13,2 / 0^{*}$ & $15,2 / 7,7^{*}$ & $15,6 / 2^{*}$ \\
\hline & Secur & $30,5 / 11,6^{*}$ & )$^{*}$ & 21 & $*$ & 19 & $18 /$ & $6,5^{*}$ & $19 / 5,4^{*}$ \\
\hline & Superiores & $42,5 / 20^{*}$ & $40,2 / 18,9^{*}$ & $35,4 / 10^{*}$ & $33,1 / 11,3^{*}$ & $30,7 / 10,7^{*}$ & $24,4 / 12,2^{*}$ & $33,1 / 10^{*}$ & $39,3 / 15,1^{*}$ \\
\hline & Sin estudios & $22,2 / 0^{*}$ & $0 / 0^{*}$ & $11,1 / 0^{*}$ & $0 / 0^{*}$ & $16,7 / 0^{*}$ & $0 / 0^{*}$ & $27,7 / 0^{*}$ & $0 / 0^{*}$ \\
\hline
\end{tabular}

\section{Conclusiones}

Siendo cierto que los alumnos autóctonos obtienen mejores calificaciones que sus iguales que han nacido en otro país, las diferencias no son estadísticamente significativas, a pesar de que estos niños presentan un perfil escolar caracterizado por una serie de variables que la investigación asocia al rendimiento educativo. Sin embargo, los datos generales del Informe PISA en sus diferentes convocatorias aluden al establecimiento de diferencias según el origen cultural de los niños.

7. Los porcentajes señalados con* corresponden a inmigrantes. 
Justamente, los datos de nuestra investigación no difieren de las conclusiones que es posible hacer para Galicia utilizando PISA-2006. En este sentido, debemos considerar que los alumnos de nuestra muestra son niños y niñas que cursan el último ciclo de Educación Primaria (fundamentalmente 6. ${ }^{\circ}$ curso) y los dos primeros cursos de ESO, mientras que el Informe PISA se centra únicamente en los alumnos de 15 años. Sin embargo, el perfil de los alumnos inmigrantes de nuestro estudio sí se caracteriza por una serie de variables que explican el bajo rendimiento de los alumnos: ser primoinmigrantes y repetidores, sobre todo en Educación Primaria.

Donde hemos podido encontrar diferencias significativas es en el nivel de estudios de los padres, ya que es más elevado entre las familias inmigrantes. Las mismas diferencias se han puesto de manifiesto a nivel intragrupal entre las familias de origen americano -mayoritarias en la muestra- y las europeas, así como entre las familias procedentes de África y las asiáticas.

Por lo tanto, si los progenitores tienen un peso tan importante en el rendimiento académico de sus hijos, será necesario adoptar estrategias de trabajo y colaboración con las familias, teniendo en cuenta sus distintas realidades educativas.

La cuestión no es baladí, ni mucho menos, sobre todo en las sociedades postindustriales, dada la importancia del éxito escolar en la integración de los inmigrantes. Lo que la actual estructura socioeconómica está provocando en miles de jóvenes es una tendencia al temprano abandono de los estudios, que les aboca al desempeño de trabajos poco cualificados, en condiciones de absoluta precariedad y con unas muy débiles expectativas de movilidad social ascendente (SuárezOrozco y Suárez-Orozco, 2008).

En el estado en el que se encuentra la gestión de la diversidad cultural en nuestro país, sometida también a los recortes presupuestarios en tanto que han afectado a importantes planes y programas en todo el abanico institucional, la cuestión del rendimiento escolar de los alumnos (autóctonos, inmigrantes asentados, inmigrantes de reciente incorporación, etc.) se ha convertido en factor de controversia en la representación de una realidad regional, nacional o incluso supranacional cuyos indicadores de equidad y cohesión social pasan hoy, inexcusablemente, por los niveles de éxito $v$ s fracaso escolar en aulas étnica y culturalmente heterogéneas.

Entre otros estudios, el Informe PISA, impulsado por un organismo de inequívoco acento economicista (OCDE), ha generado interesantes activos para un mejor conocimiento del cuadro que resulta en nuestras escuelas cuando se hacen comparaciones del tipo que permite hacer este documento de indagación trianual (alumnos autóctonos-alumnos de origen inmigrante) alrededor de un amplio abanico de variables sociofamiliares y circunstancias académicas. Y aunque España sea uno de los países, junto a Finlandia, con un mayor nivel de equidad, ello no quiere ni puede significar la ausencia de diferencias entre las CC. AA. Que no se pueden explicar simplemente en función de los contingentes o de la presión migratoria hacia distintas áreas y entornos del país. 


\section{REFERENCIAS BIBLIOGRÁFICAS}

Calero, J.; Choi, A. y Waisgrais, S. (2009) Determinantes del rendimiento educativo del alumnado de origen nacional e inmigrante en PISA-2006. Información Comercial Española, 78, 281-310.

- (2010) ¿Qué determina el fracaso escolar en España? Un estudio a través de PISA-2006. Revista de Educación, n. ${ }^{\circ}$ ext., 225-256.

CARABAÑa, J. (2008) El impacto de la inmigración en el sistema educativo español. ARI, n. ${ }^{\circ}$ 63/2008. Área: Demografía, Población y Migraciones Internacionales. Real Instituto Elcano. Consultado el 20 de febrero de 2011. http://www.realinstitutoelcano.org/wps/ portal/rielcano/contenido?WCM_GLOBAL_CONTEXT=/elcano/elcano_es/programas/ migraciones+internacionales/publicaciones/ari63-2008.

Cebolla, H. (2009) Inmigración y educación: del shock demográfico al debate sobre el rendimiento, en Cebolla, H. y LaRios, M. ${ }^{a}$ J. (eds.) Inmigración y educación. Madrid, Centro de Estudios Políticos y Constitucionales, 9-55.

CIDE (2005) La atención al alumnado inmigrante en el sistema educativo en España. Madrid, CIDE.

Dronkers, J. (2010) Influència dels països d'origen i de destí en el rendiment de l'alumnat d'origen immigrat. Debats d'Educació, 17. Barcelona, Fundació Jaume Bofill.

EUROPEAN COMMISSION (2008) Education and migration. Strategies for integrating migrant children in European schools and societies. Bruselas, European Commission.

Fernández Enguita, M.; Mena MarTínez, L. y Riviere GÓmez, J. (2010) Fracaso y abandono escolar en España. Barcelona, Fundación "La Caixa”.

FERRER, F. et al. (2008) Equitat, eficiencia i excellència educativa a Catalunya. Una anàlisi comparada. Barcelona, Fundación Jaume Bofill.

GutiÉRREZ-DomĖNech, M. (2009) Factors determinants del rendiment educatiu: el cas de Catalunya. Documents d'Economia "La Caixa", n. ${ }^{\circ} 15$. Barcelona, "la Caixa".

INCE (1968-1997) Elementos para un diagnóstico del sistema educativo español. Madrid, INCE.

InSTITUTO DE EVALUACIÓn (2010a) PISA 2009. Programa para la Evaluación Internacional de los Alumnos. Informe español. Madrid, Ministerio de Educación.

- (2010b) Evaluación General de Diagnóstico 2009. Educación Primaria. Cuarto Curso. Informe de Resultados. Madrid, Ministerio de Educación.

Krueger, R. A. (1991) El grupo de discusión. Guía práctica para la investigación aplicada. Madrid, Pirámide.

OCDE (2006) PISA 2006. Marco de la evaluación. Conocimientos y habilidades en ciencias, matemáticas y lectura. París, OCDE.

PEDRó, F. (2009) Las políticas educativas sobre migración: una visión comparativa, en Martínez Usarralde, M. ${ }^{a}$ J. (ed.) Educación internacional. Valencia, Tirant lo Blanch, 153-193.

Santos Rego, M. A. (dir.) (2010) O rendemento escolar do alumnado procedente da inmigración en Galicia. Un programa de optimización para familias e centros de ensino. Informe de Investigación (inédito).

Santos Rego, M. A.; Crespo Comesaña, J.; lorenzo Moledo, M. y Godás Otero, A. (2011) Escuelas e Inmigración en España. ¿Es inevitable la segregación? Cultura y Educación (en prensa). 
SERra, C. y PALAudÀries, J. M. (2007) L'alumnat de nacionalitat estrangera en els estudis posobligatoris, en LARIOS, M. ${ }^{a} \mathrm{~J}$. y NADAL, M. (eds.) L'estat de la immigració a Catalunya (Anuari 2006). Barcelona, Mediterrània, 301-334.

SHAFFER, R. D. (2002) Desarrollo social y de la personalidad. Madrid, Thomson Editores. SíNDIC DE GREUGES (2008) La segregación escolar en Cataluña. Barcelona, Síndic de Greuges.

Sotelino, A. y VARELA, C. (2011). Convivencia y diversidad cultural en los centros educativos gallegos. Comunicación presentada al II Congreso Nacional sobre convivencia y resolución de conflictos en contextos socioeducativos. Málaga, 24-26 de marzo.

SuÁrez-Orozco, C. y SuÁrez-Orozco, M. (2004) La infancia de la inmigración. Madrid, Morata.

- (2008) Històries d'immigració: la comprensió dels patrons de rendiment escolar dels joves immigrants. Barcelona, Fundació Jaume Bofill. 